Research Article

\title{
Effect of Properties of Silver Nanoparticles Coated with Polar Material and the Antibacterial Activity on Marine Pathogenic Bacteria
}

\author{
Yanli Feng, ${ }^{1,2}$ Tian Deng,, ${ }^{1,2}$ Xiaohua Lai, ${ }^{1,2}$ Zhihua Feng, ${ }^{1,2}$ Mingsheng Lyu $\mathbb{D}^{1,2}$ \\ and Shujun Wang $\mathbb{1}^{1,2}$ \\ ${ }^{1}$ Jiangsu Key Laboratory of Marine Bioresources and Environment/Jiangsu Key Laboratory of Marine Biotechnology, \\ Jiangsu Ocean University, Lianyungang 222005, China \\ ${ }^{2}$ Co-Innovation Center of Jiangsu Marine Bio-Industry Technology, Jiangsu Ocean University, Lianyungang 222005, China
}

Correspondence should be addressed to Mingsheng Lyu; mingshenglu@hotmail.com and Shujun Wang; shujunwang86@163.com

Received 17 April 2020; Revised 25 August 2020; Accepted 29 October 2020; Published 24 November 2020

Academic Editor: Thathan Premkumar

Copyright (c) 2020 Yanli Feng et al. This is an open access article distributed under the Creative Commons Attribution License, which permits unrestricted use, distribution, and reproduction in any medium, provided the original work is properly cited.

\begin{abstract}
Silver nanoparticles (Ag NPs) have become one of the current research hotspots and are used in many fields such as electrochemistry, energy, bioanalysis, and environmental monitoring, especially in the field of antibacterial research. In this study, we investigated the effect of properties of Ag NPs coated with polar materials. Ag NPs covered by a dispersant that was triethylene glycol monoethyl ether was stable and conquered the aggregation of Ag NPs. The effect of the dispersant on biocompatibility was explored through interaction experiments between Ag NPs and DNA sequence. The coated Ag NPs could adsorb DNA, and the fluorescence of FAM-DNA could be quenched by Ag NPs. The adsorption and desorption experiments of DNA showed that the order of DNA functional groups on the interaction process was phosphate $>\mathrm{T}>\mathrm{C}>\mathrm{A}>\mathrm{G}$. Moreover, we selected marine pathogenic bacteria to test the antibacterial effect of Ag NPs coated with a polar dispersant. The polar material had a certain inhibitory effect on the antibacterial activity of Ag NPs. However, small molecules such as bases could interact on the surface Ag NPs and release $\mathrm{Ag}^{+}$to perform the antibacterial activity. The results could contribute to the further application of Ag NPs.
\end{abstract}

\section{Introduction}

Silver nanoparticles (Ag NPs) have attracted significant attention based on their excellent properties [1]. Silver is a good conductor and resistant to oxidation and has been used in printed conductors and printable inks. Ag NPs with broad-spectrum antibacterial properties are natural inorganic bactericides [2]. Salem and his colleagues found that Ag NPs have good resistance to Vibrio cholerae and Escherichia coli that causes bowel disease [3]. Panáček's group determined that the minimum inhibitory concentration (MIC) of Ag NPs on fungal pathogen Candida spp. was $0.05 \mathrm{mg} / \mathrm{L}$ and that the concentration had no significant inhibitory effect on the growth of human fibroblasts [4]. Elechiguerra's group found that Ag NPs interact with HIV-1, and this interaction process inhibits its binding to host cells [5]. Gurunathan's group reported the inhibitory effect of Ag NPs on Gramnegative and Gram-positive bacteria [6]. The abuse of antibiotics caused drug resistance in many strains, but most of them did not show resistance to Ag NPs. Therefore, Ag NPs have been widely used as an antibacterial material in wound dressings $[7,8]$, medical equipment [9-11], food packaging [12], textiles [13, 14], and air purifiers [15].

Ag NPs have good biocompatibility and can be assembled with biological macromolecules such as DNA, proteins, and carbohydrates $[16,17]$. In Zhao et al.'s research, DNA$\mathrm{Ag}$ was used as a temperature-sensitive carrier for drug loading. On the duplex, the drug load per CG pair is 1 doxorubicin (Dox) molecule [18]. Loaded Dox can be released by increasing the melting temperature of the stem duplex. In addition, special DNA sequences make it sensitive to HepG-2 cells [18]. Ag NPs have been developed as biosensors 
TABLE 1: DNA used in the experiment.

\begin{tabular}{|c|c|}
\hline Name & Sequence $\left(5^{\prime} \rightarrow 3^{\prime}\right)$ and modification \\
\hline FAM-A15 & 6 FAM-AAAAAAAAAAAAAAA \\
\hline FAM-T15 & 6 FAM-TTTTTTTTTTTTTTT \\
\hline FAM-C15 & 6 FAM-ССССССССССССССС \\
\hline FAM-G15 & 6 FAM-GGGGGGGGGGGGGGG \\
\hline FAM- $A_{5}$ & 6 FAM-AAAAA \\
\hline FAM- $\mathrm{A}_{30}$ & 6 FAM-AAAAAAAAAAAAAAAAAAAAAAAAAAAAAA \\
\hline FAM- $\mathrm{A}_{45}$ & $\begin{array}{c}\text { 6 FAM-AAAAAAAAAAAAAAAAAAAAAAA } \\
\text { AAAAAAAAAAAAAAAAAAAAAA }\end{array}$ \\
\hline FAM-24mer & 6 FAM-ACGCATCTGTGAAGAGAACCTGGG \\
\hline
\end{tabular}

[19] and drug carriers [20] and have been applied in popular areas such as diagnosis and treatment of diseases [21,22], biological detection [23], and environmental analysis [24].

At present, in the field of antibacterial research, there are a wide variety of antibacterial materials and advanced antibacterial technologies. Zhang and his research team discovered that copper $(\mathrm{Cu})$ could be used in biomedical materials [25], because $\mathrm{Cu}$ could exert its alloy functions such as corrosion resistance and wear resistance, as well as good antibacterial and biological activity. However, the copper content is insufficient and excess can cause harm to humans. In addition, Zhang and his colleagues incorporated copper into the atomic nickel-titanium alloy used in to improve its antibacterial effect [26]. Huang's research group simulated click chemistry to construct a paste xerogel as a portable treatment for bacterial wounds. Sulfuric acid-based gel can absorb $60 \%$ of bacterial infections under Staphylococcus aureus through static electricity [27]. But this was only suitable for dealing with emergency infections, unlike silver nanomaterials which have general applicability. With the development of optical technology, more advanced photocatalytic antibacterial technology, photothermal spectroscopy, and other antibacterial methods have gradually emerged. Luo et al.'s subject combined into a core-shell bimetallic organic framework heterogeneous interface. Porphyrin could enhance the transfer of $\mathrm{PB}$ light-excited electrons and improve the photocatalytic performance. Under $660 \mathrm{~nm}$ light, high concentration of singlet oxygen is generated to achieve antibacterial effect [28]. Li's research group constructed a hybrid nanosheet $\left(\mathrm{g}-\mathrm{C}_{3} \mathrm{~N}_{4}-\mathrm{Zn}^{2+} @ \mathrm{GO}(\mathrm{SCN}\right.$ $\left.\mathrm{Zn}^{2+} @ \mathrm{GO}\right)$ ) doped with zinc ions. The technology achieved rapid sterilization and wound healing by exposure to light $(660 \mathrm{~nm}$ and $808 \mathrm{~nm})$ in a short time through electrostatic bonding and $\pi-\pi$ stacking interaction [29]. The mechanism may be that the high-temperature effect of $808 \mathrm{~nm}$ light could affect the metabolism of bacteria. Furthermore, active oxygen was generated under $660 \mathrm{~nm}$ light, which could destroy cell membranes, denature bacterial proteins, destroy bacterial metabolism, and achieve antibacterial effect.

Silver nanoparticles have the advantages of broadspectrum antibacterial property, strong sterilization, simple preparation, little harm to the environment, and resistance to drug resistance. It was widely used as an antimicrobial material for various products. The antibacterial effects of nanosilver mainly included affecting the living environment of bacteria, destroying the cell wall structure of bacteria, inhibiting activity of enzymes, and interacting with bacterial DNA and inhibiting its replication. The antibacterial process of silver nanoparticles was often very complicated, and the process was affected by many factors. Hu et al.'s research had demonstrated that Ag NPs could be etched by DNA and release $\mathrm{Ag}^{+}$. Also, the cytosine was most efficient for the activity of etching [30]. Actually, the $\mathrm{Ag}^{+}$was the main ion to kill bacteria. However, Ag NPs have poor stability and are easy to agglomerate [31]. Moreover, the surface of $\mathrm{Ag}$ NPs would adsorb different polar molecules when exposed in the environment based on the nanosilver surface with positive charge. So, it is meaningful to investigate the properties of Ag NPs coated with polar materials.

When Ag NPs were used in an organism to perform antimicrobial activity, Ag NPs would interact with macromolecules that are polar materials mainly. We hope to investigate the effects for the Ag NP characteristics coated with a polar material. In this study, we focused on the property of Ag NPs which were coated with polar materials. We chose commercial Ag NPs that were dispersed in the triethylene glycol monoethyl ether. We expected to investigate the effect of the property of Ag NPs. As Ag NPs could be etched by DNA, we explored the relationship between Ag NPs and DNA and the effect on the antibacterial activity of Ag NPs.

\section{Materials and Methods}

2.1. Materials. Ag NPs ( $\leq 50 \mathrm{~nm})$, adenine, guanine, cytosine, and thymine reagents were purchased from Sigma-Aldrich. All desalted purified oligonucleotide sequences (Table 1) were purchased from Shanghai Sangon Biotech. And all of the DNA sequences were labeled by fluorescence. Silver nitrate $\left(\mathrm{AgNO}_{3}\right)$ reagent and analytical grades of glucose and fructose reagents were purchased from Sinopharm Group. Polystyrene 96-well plates were purchased from Corning, Inc. The water used in the experiment was ultrapure water $(18.0 \mathrm{M} \Omega)$. Silver nanoparticles used in this experiment have been coated with triethylene glycol monoethyl ether when we bought them.

\subsection{Characterization of $\mathrm{Ag} \mathrm{NPS}$}

2.2.1. Transmission Electron Microscopy. The morphology of the Ag NPs was studied using transmission electron 


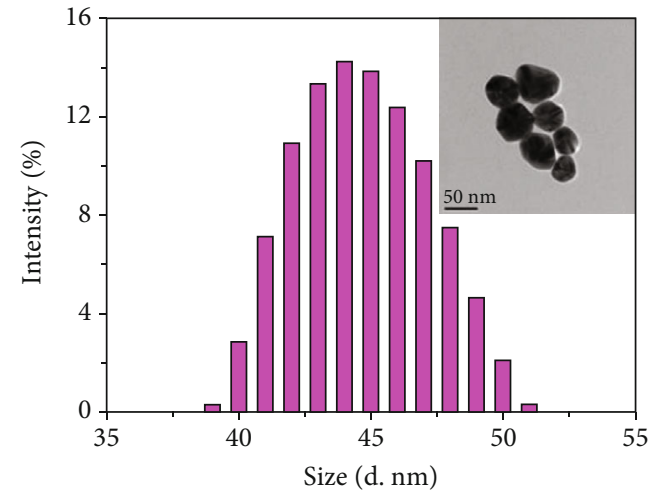

(a)

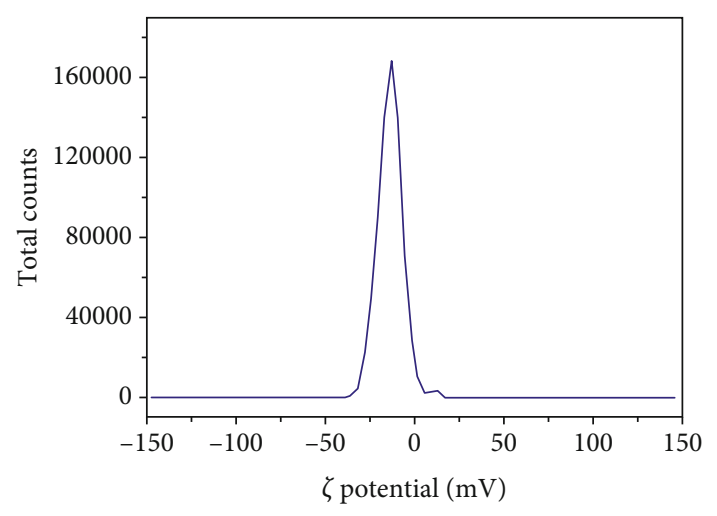

(b)

Figure 1: Physical characterizations of Ag NPs by (a) DLS (45 $\pm 7 \mathrm{~nm})$ and TEM (inset) and (b) $\zeta$ potential.

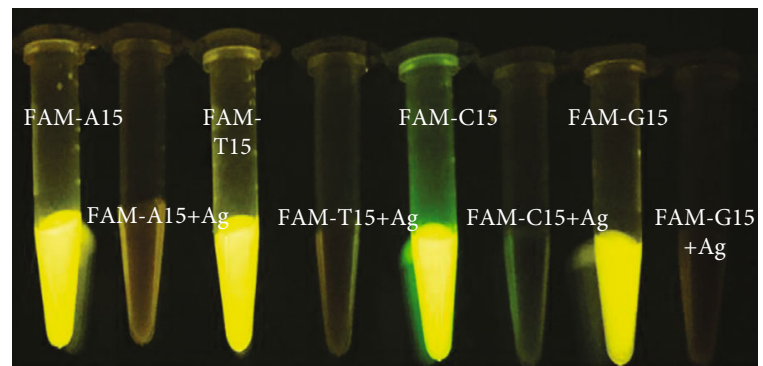

(a)

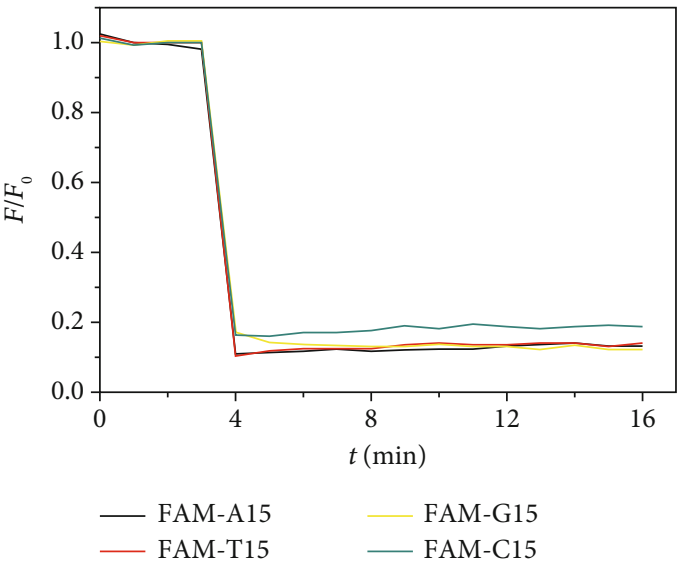

(c)

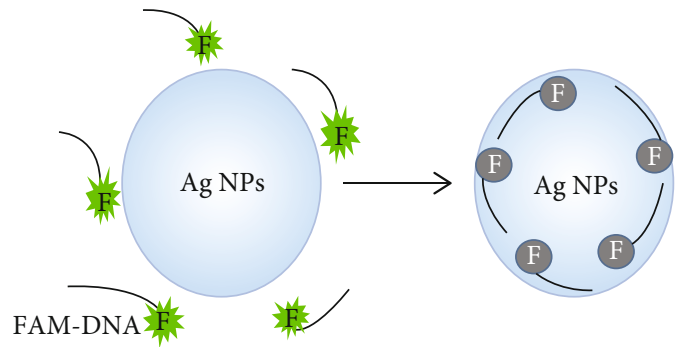

(b)

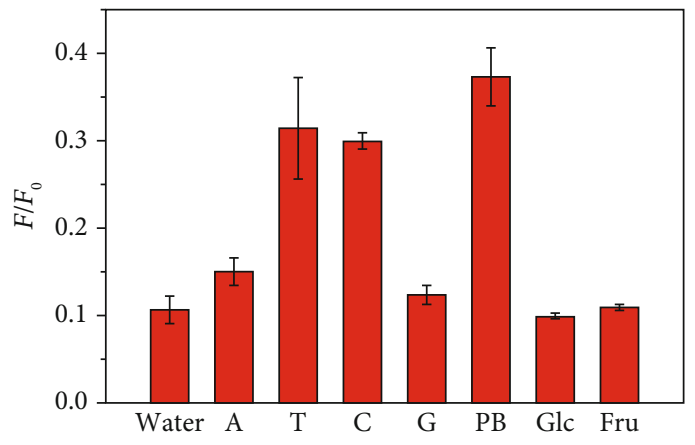

(d)

Figure 2: (a) Ag NPs quench the fluorescence of FAM-DNA upon adsorption. (b) Scheme showing adsorption of FAM-labeled DNA with fluorescence quenched. (c) Fluorescent kinetic spectrograms of the interaction between Ag NPs and DNA. (d) Effect of bases, phosphate buffer, and glucose/fructose on the adsorption of DNA on Ag NPs.

microscopy (TEM, Philips CM 10) after drying the particle dispersions on a holey carbon copper grid.

\subsubsection{Fourier Transform Infrared Spectrometer. The Ag NPs} diluted with ultrapure water and the nanosilver-DNA conjugate solution and potassium bromide were dried and reserved. In the sample preparation process, about $300 \mathrm{mg}$ potassium bromide was added. The samples were selected by capillary tube and grinded. Then, a pill was made by pressing and determined by Fourier transform infrared (FT-IR) spectroscopy.
2.2.3. Dynamic Light Scattering. The size and surface potential of Ag NPs were measured with a Zetasizer Nano particle size analyzer (Malvern, London, UK). The Ag NPs were diluted with $2 \mu \mathrm{M}$ ultrapure water. $3 \mathrm{~mL}$ of the above samples was taken for detection. The concentration of Ag NPs in $\zeta$ potential was $0.2 \mathrm{mM}$.

2.2.4. X-Ray Diffraction Analysis. The powder XRD patterns of various samples were collected on a Bruker D8 diffractometer using $\mathrm{Cu} \mathrm{K} \alpha$ radiation (Bruker, Germany). The pattern 


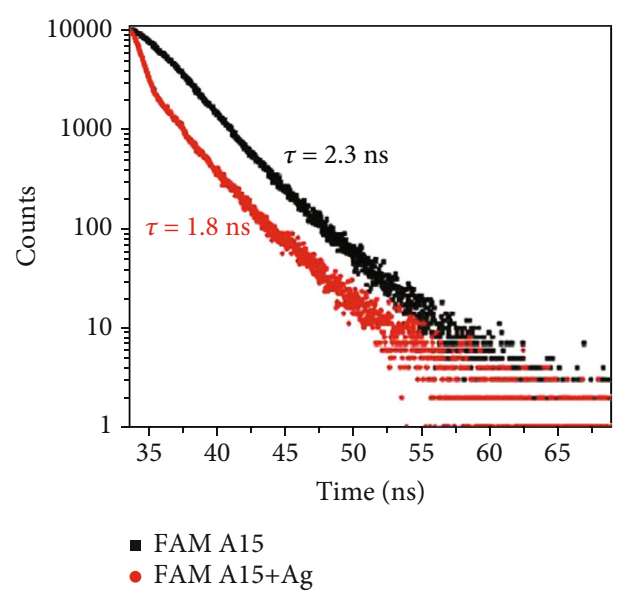

(a)

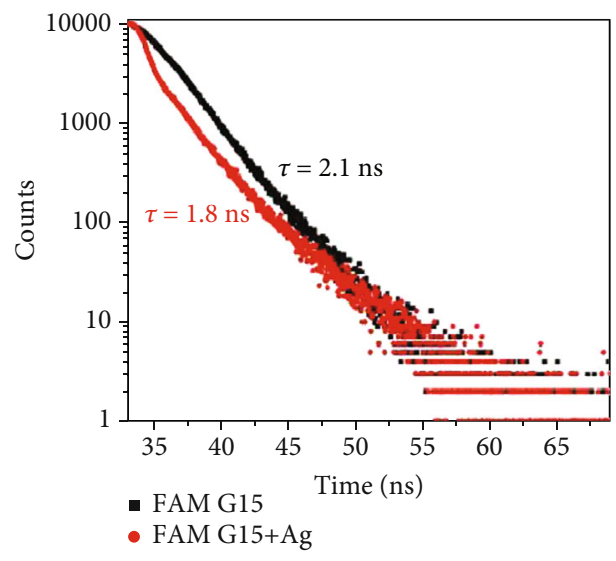

(c)

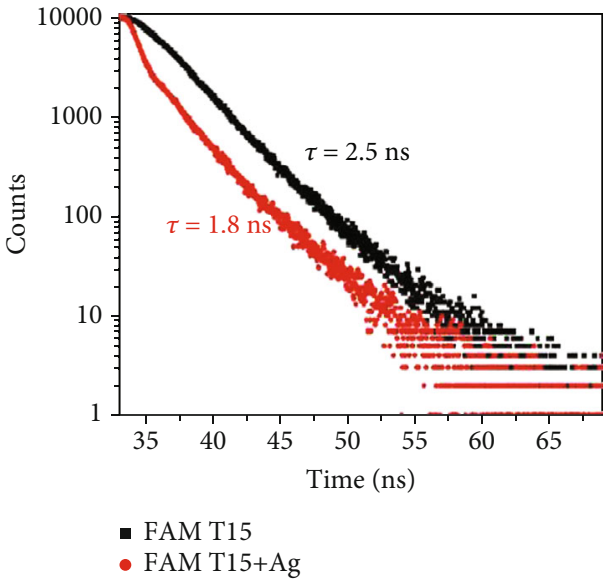

(b)

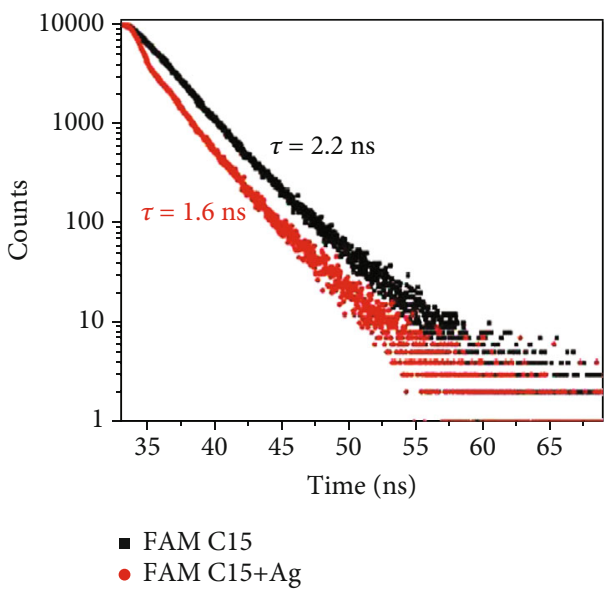

(d)

Figure 3: The fluorescence lifetime decay. (a) FAM-A15 and FAM-A15 with Ag NP. (b) FAM-T15 and FAM-T15 with Ag NP. (c) FAM-G15 and FAM-G15 with Ag NP. (d) FAM-G15 and FAM-C15 with Ag NP.

was recorded over the $5-90^{\circ} 2 \theta$ range in steps of $0.05^{\circ}$ with a counting time per step of $1 \mathrm{sec}$.

2.3. Interaction between Ag NPs and DNA. DNA labeled with fluorescence was prepared into a $100 \mu \mathrm{M}$ reserve solution in ultrapure water and stored at $-20^{\circ} \mathrm{C}$. The experiments selected have four 6-carboxyfluorescein-labeled DNA, namely, FAM-A15, FAM-T15, FAM-C15, and FAM-G15. Firstly, 10 nM DNA samples were added to a 96-well plate, and then, the fluorescent signal was detected for $3 \mathrm{~min}$ in the multifunctional microplate reader kinetic mode. The fluorescence spectrum of the interaction between Ag NPs and DNA was recorded by the M2000 multifunctional microplate reader (Tecan, Switzerland). The excitation wavelength was $485 \mathrm{~nm}$, and the emission wavelength was $525 \mathrm{~nm}$. The obtained fluorescence signal value was the initial fluorescence signal value. Subsequently, $1 \mathrm{mM} \mathrm{Ag} \mathrm{NPs} \mathrm{was} \mathrm{added}$ and detected continually for $12 \mathrm{~min}$.

Furthermore, we explored the effects of various functional groups of DNA on the interaction between Ag NPs and DNA. The part of experiment was explored by competitive adsorption experiments. In the experiments, we added
FAM-24mer sequence and Ag NPs in solution, thoroughly mixed, and incubated for $15 \mathrm{~min}$ at room temperature. Hereafter, substitutes for various functional groups of DNA such as phosphate buffer, four nucleotide base, glucose, and fructose were added, respectively. Finally, we used the microplate reader to record the fluorescence signal.

2.4. Inhibitory Activity of Ag NPs on Marine Pathogenic Bacteria. The Oxford cup and spread-plate method was used to determine the inhibitory effect of the Ag NPs for the growth of marine bacteria $V$. anguillarum and Edwardsiella tarda. The bacteria strains were inoculated in LB medium and cultured 16 hours at $30^{\circ} \mathrm{C}$ and $180 \mathrm{rpm}$. We took the activated bacterial solution and diluted it to $10^{-3}$ serially as seed solution. Then, we took $100 \mu \mathrm{L}$ seed solution and spread on plates of LB medium. $150 \mu \mathrm{L}$ of samples was added in the Oxford cups, respectively. At the same time, the same volume of $75 \%$ ethanol was used as the positive control. Finally, the plates were placed in the incubator at $30^{\circ} \mathrm{C}$ for 24 hours. The broth was detected at $600 \mathrm{~nm}$ by a spectrophotometer. 


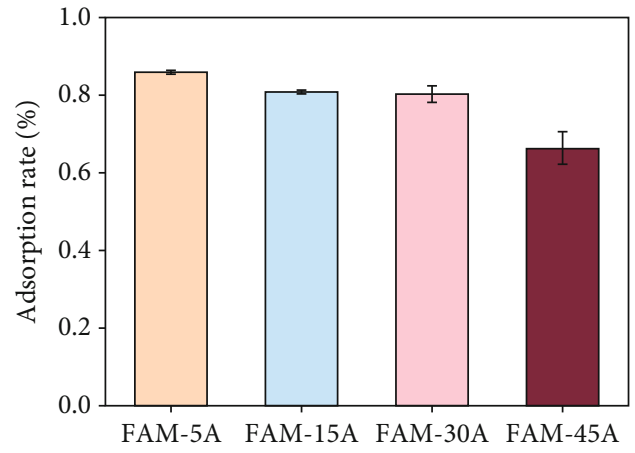

(a)

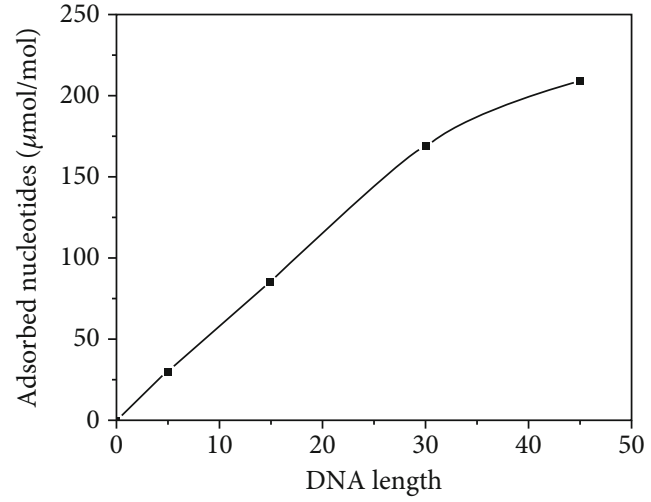

(b)

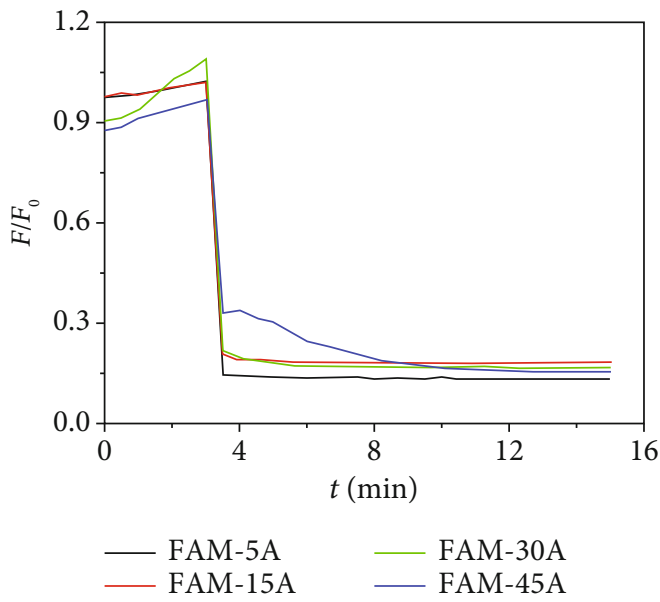

(c)

Figure 4: (a) Effect of DNA length on the adsorption capacity of DNA. (b) Number of nucleotide footprints on Ag NP surfaces. (c) DNA length effect on DNA adsorption kinetics.

\section{Results and Discussion}

3.1. Characterization of Ag NPs. According to dynamic light scattering (DLS) measurement, the average particle size of the Ag NPs was about $45 \pm 7 \mathrm{~nm}$. Most of the silver nanoparticles were spherical particles with uniform shape (Figure 1(a)). The particle size distribution was relatively concentrated. Along with the experiments, the Ag NP solution and the diluted solution presented very stable. As shown in Figure 1(b), Ag NPs are negatively charged in ultrapure water. The zeta potential of Ag NPs was $-13.7 \mathrm{mV}$. Since the triethylene glycol monoethyl ether dispersant was slightly negatively charged after dilution with ultrapure water, it suggested that the Ag NPs were coated with the polar dispersant.

3.2. Interaction between $A g N P s$ and DNA. The fluorescence signal was quenched, so an electron transfer occurred during the interaction of Ag NPs with FAM-DNA (Figure 2(a)). As shown in Figure 2(b), FAM-DNA combines with Ag NPs, so the fluorescence of DNA was quenched. According to the fluorescence kinetic spectra of Ag NPs with FAM-DNA (Figure 2(c)), the fluorescence signal value decreases in a short time, which indicates that the interaction process between them was extremely fast. It also suggested that all the four kinds of oligonucleotide sequences (FAM-A15, FAM-T15, FAM-C15, and FAM-G15) could combine with the Ag NPs.

DNA is an organic macromolecule composed of deoxyribonucleotides. The three parts of the phosphate backbone, the nitrogen-containing bases (adenine, thymine, cytosine, and guanine) and deoxyribose constitute the deoxyribonucleotides. We investigated the effect of analogs of DNA functional groups between Ag NPs and DNA. We selected their analogs. The inorganic phosphate buffer solution replaced the phosphate backbone, and fructose and glucose replaced the ribose. A random sequence of FAM-24mer nucleotides was used. As Figure 2(d) shows, the effect of the phosphate buffer solution and the four nucleotide bases on the interaction process is different, and the order of the impact strength was phosphate $>\mathrm{T}>\mathrm{C}>\mathrm{A}>\mathrm{G}$. The effect of fructose and glucose on the interaction process was particularly small. In other words, deoxyribose might have little effect on the interaction process between Ag NPs and DNA. Notably, the phosphate backbone played a dominant role, and thymine and cytosine were more affinities than adenine and guanine with Ag NPs.

Figure 3 shows the fluorescence lifetime of the labeled DNA and Ag NPs mixed with labeled DNA. All the 

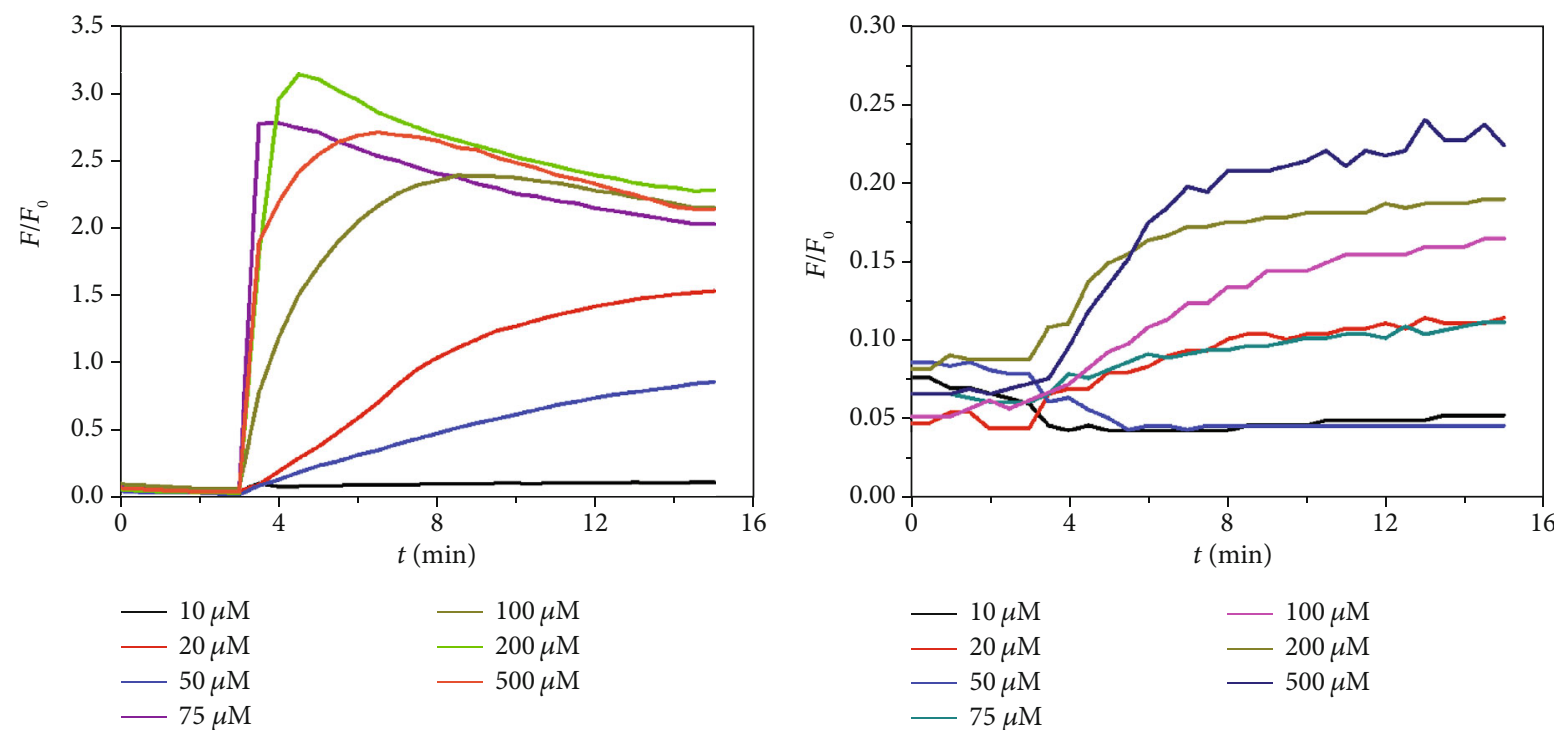

(a)

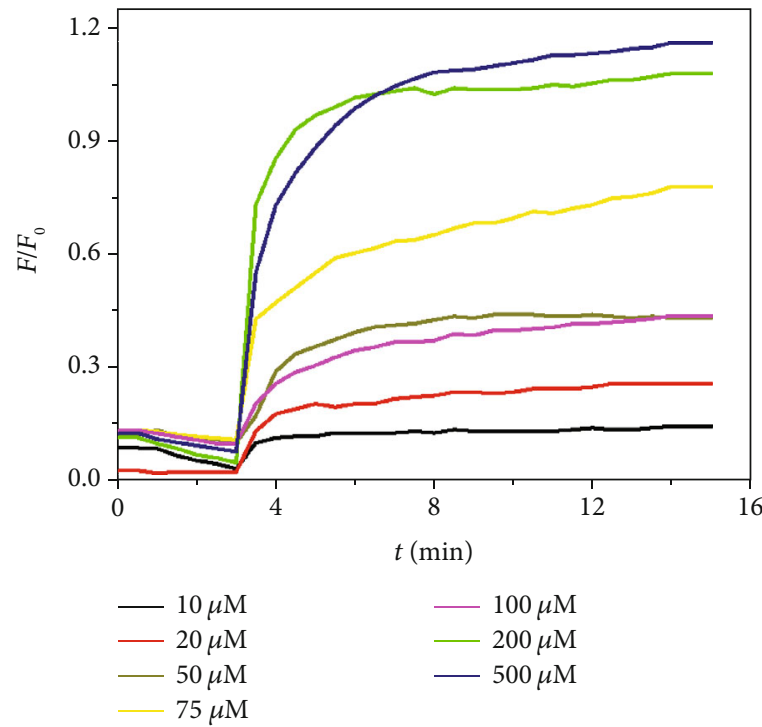

(c)

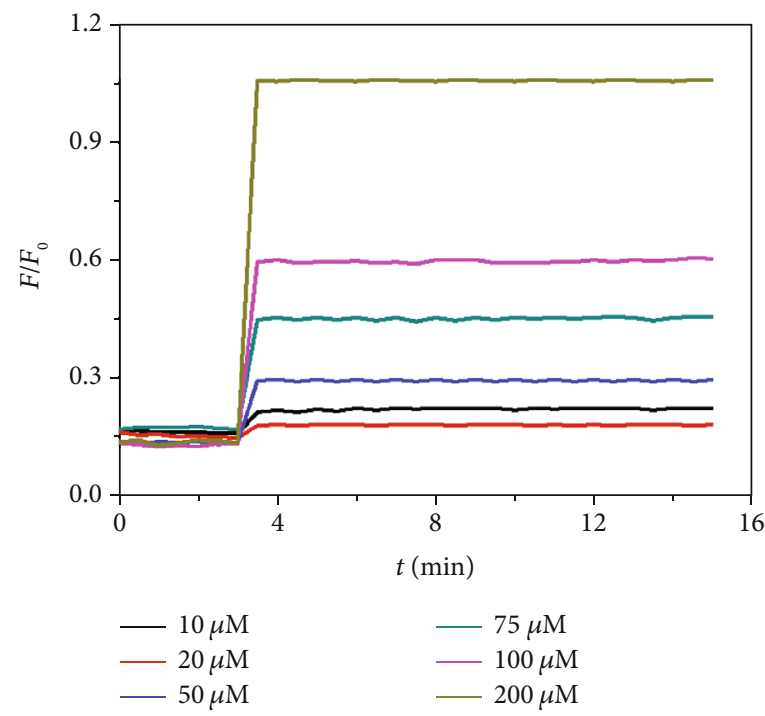

(d)

Figure 5: The effect of DNA desorption from Ag NPs by various concentrations of cytosine: (a) FAM-A15, (b) FAM-T15, (c) FAM-C15, and (d) FAM-G15.

fluorescence lifetime of the mixture was decreased in a short time which demonstrated the combination of Ag NPs and DNA [32]. Moreover, we detected the mixture by TEM, and the Ag NPs aggregated in all four different bases singlestrand DNA. As showing in Figure S1, Ag NPs aggregate most significantly after adsorption of FAM-C15, followed by FAM-A15. The results suggested that the surface charge of Ag NPs was changed by DNA. During the experiment, we found that Ag NPs interacted with DNA and aggregated easily. We could found precipitation in EP tubes. Zhang et al.'s research reported the same phenomenon when Au NPs interacted with thiolate DNA [33].

In the process of Ag NP adsorption of DNA, the phosphate backbone plays a major role. It may be that the phosphate backbone of the DNA forms a chemical coordinate bond with Ag NPs. The surface of the Ag NPs is positively charged while the phosphate backbone is negatively charged. Therefore, they have a strong electrostatic effect between them. Four nucleic bases also promote the adsorption, of which thymine has the greatest influence on adsorption. Ribose has a little effect on adsorption.

It was initially speculated that an $\mathrm{N}-\mathrm{H}$ hydrogen bond was formed between the Ag NPs and the base. In order to verify this hypothesis, we performed an elution experiment of DNA adsorbed by Ag NPs in $1 \mathrm{M}$ urea solution. It was found that there was no elution effect.

The different lengths of DNA were adsorbed in different quantities on the surface of Ag NPs. The results suggested that the DNA was bent on the Ag NPs (Figure 4).

3.3. DNA Desorption. After adsorption of fluorescently labeled DNA by Ag NPs, the addition of inorganic phosphate 


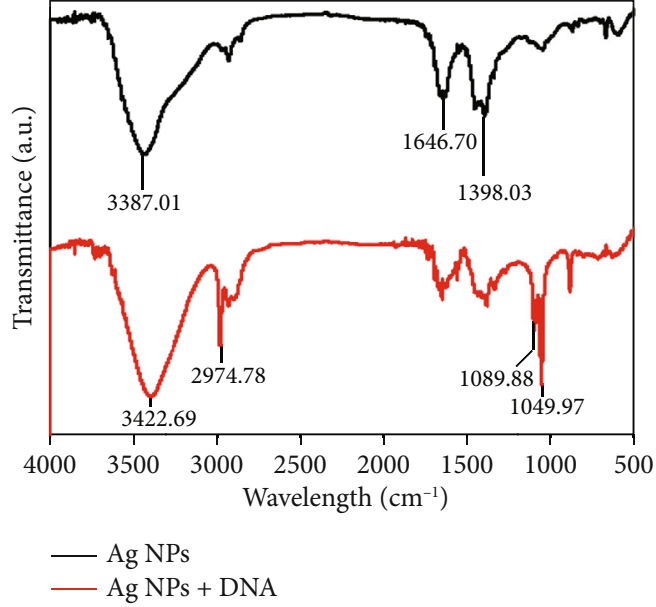

(a)

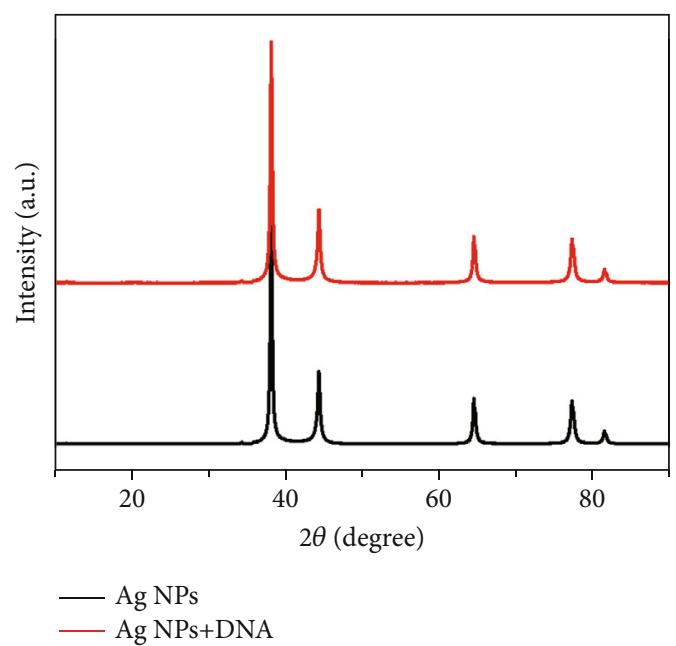

(b)

FIGURE 6: (a) FT-IR and (b) XRD spectra before and after adsorption of DNA by Ag NPs.

buffer and nucleobases could induce DNA desorption. As the concentration of cytosine increased, the amount of desorbed DNA increased. We conducted the experiments on Ag NPs with FAM-A15, FAM-T15, FAM-C15, and FAM-G15 (Figure 5). Comparing the four DNA sequences, FAM-T15 was the most difficult to desorb. It is suggested that Ag NPs and FAM-T15 have the strongest adsorption effect. DNA desorption was more pronounced when the free cytosine was added nearly 1000 times higher than the FAM-15C (i.e., $10 \mu \mathrm{M}$ free cytosine concentration and $10 \mathrm{nM}$ FAM15C). There was a strong interaction between the base of DNA and Ag NPs. It was inferred that strong interaction forces were physical adsorption because it was irreversible.

The experiments of desorption by phosphate buffer (Figure S2), adenine (Figure S3), thymine (Figure S4), and guanine (Figure S5) were also performed, and the results were similar.

3.4. Mechanism of DNA Adsorption. Comparing the FT-IR spectra of Ag NPs and Ag NP-adsorbed DNA, there was hydrogen bond between Ag NPs and DNA (Figure 6(a)). Metals do not have absorption peaks in the infrared spectrum, and even metal nanoparticle infrared light could not be transmitted. Therefore, the characteristic peak of the infrared spectrum labeled as Ag NPs was a peak of triethylene glycol monoethyl ether. The characteristic peaks of the infrared spectrum of Ag NPs and DNA conjugates were characteristic peaks of DNA and polar solvents. Broadband approximately at $3387.01 \mathrm{~cm}^{-1}$ and $3422.69 \mathrm{~cm}^{-1}$ may be due to $-\mathrm{OH}$ tensile vibration. The weak absorption peak at $1646.70 \mathrm{~cm}^{-1}$ was the characteristic peak of $\mathrm{C}=\mathrm{O}$. We speculated that the C-O electron cloud in triethylene glycol monoethyl ether overlaps. The absorption peak at a bandwidth of $1398.03 \mathrm{~cm}^{-1}$ was due to the bending vibration of $\mathrm{C}-\mathrm{H}$. It is generally accepted that DNA was an organic macromolecule. In the infrared spectrum of Ag NPs and DNA conjugate, the peak with a bandwidth of $2974.78 \mathrm{~cm}^{-1}$ was $\mathrm{C}-\mathrm{H}$ in $-\mathrm{CH}_{2}$, and the absorption peak with a bandwidth of $1089.88 \mathrm{~cm}^{-1}$ was a $\mathrm{C}-\mathrm{O}$ bond. The bandwidth of $1049.97 \mathrm{~cm}^{-1}$ was the character- istic peak of the $\mathrm{C}-\mathrm{N}$ bond. From the XRD spectrum (Figure 6(b)), the characteristic peaks of Ag NPs were consistent before and after DNA adsorption. Figure 6(b) shows that the crystal structure of Ag NPs does not change before and after DNA adsorption.

3.5. Effect of the Antibacterial Activity. In the experiments, three marine pathogenic bacteria $V$. anguillarum and E. tarda were selected to investigate the effect of polar dispersant on the antibacterial properties of Ag NPs. The antibacterial activity of $5 \mathrm{mM}$ Ag NPs and Ag NPs-DNA conjugates against $V$. anguillarum was determined by the Oxford cup method (Figure 7(a)). Neither Ag NPs nor the Ag NPsDNA conjugate produced an obvious zone of inhibition on the plate. There was a relatively obvious antibacterial activity when the concentration of Ag NPs was $10 \mathrm{mM}$ only (Figure 7(b)). The results show in Figure $7(\mathrm{c})$ that there is basically no difference in the effect of Ag NPs and DNA conjugates on the growth of E. tarda. However, the base-Ag NP combination had a relatively significant effect, especially adenine. The order of influence was $A>T>C$. There was a speculation that Ag NPs interact with bases to release more silver ions, which significantly enhances antibacterial activity. In Figure 7(d), in the spread method of E. tarda, we can see that Ag NPs coated with polar dispersant do not show the antibacterial activity. The Ag NPs and the mixture of Ag NPs with DNA performed the same with the control. The polar dispersant had inhibited the release of $\mathrm{Ag}^{+}$.

There is no denying that Ag NPs have a unique broadspectrum antibacterial property (Table S1). The sterilization effect of Ag NPs is affected by various factors such as particle size, shape, surface potential, coating reagent, and dispersant. Generally, the smaller the particle size, the better the antibacterial effect of the positively charged Ag NPs on the surface [34]. Our results showed that Ag NPs coated with triethylene glycol monoethyl ether almost lose their antibacterial effect except on much higher concentration (Table S1). The polar dispersant had an inhibitory effect on the antibacterial properties of Ag NPs. 


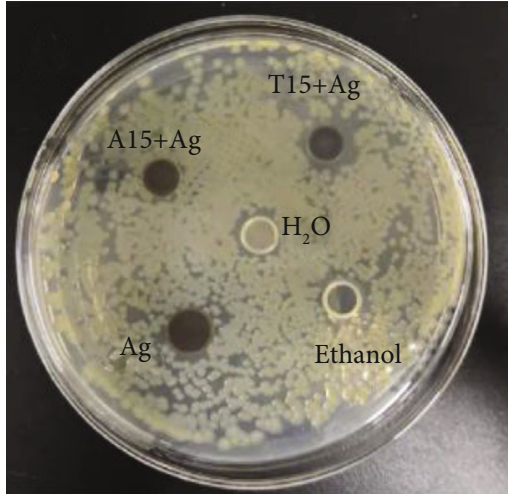

(a)

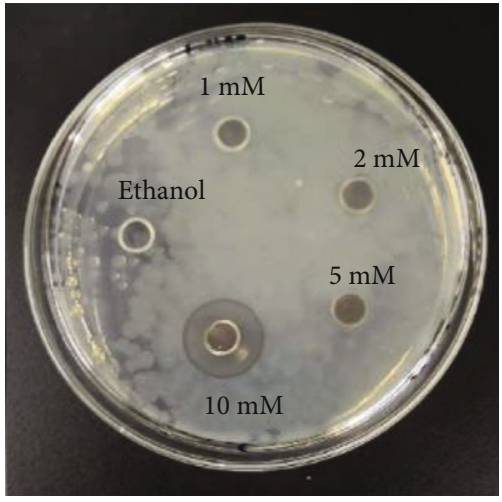

(b)
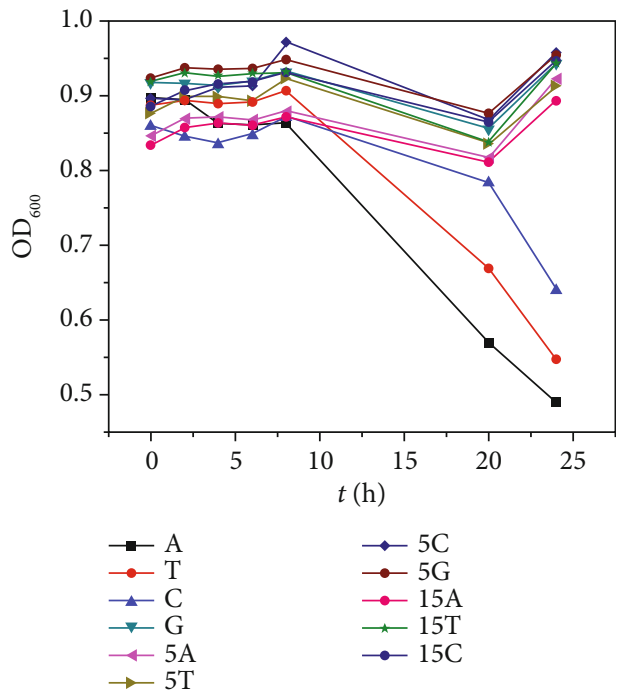

(c)

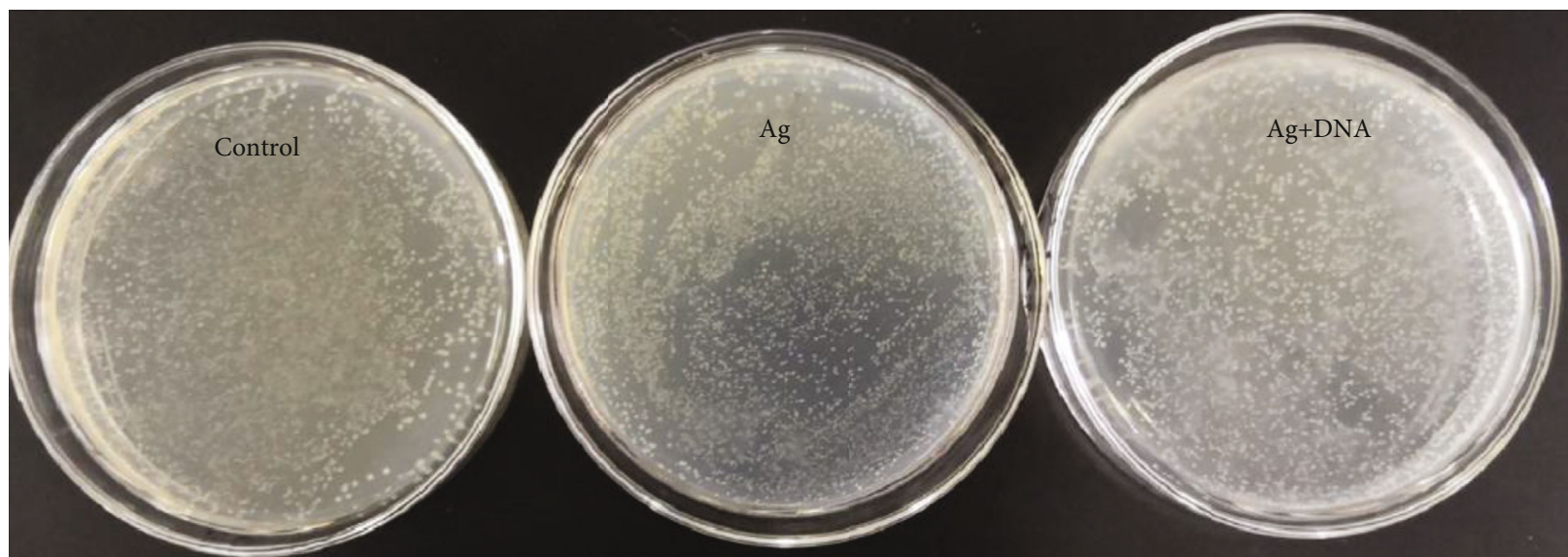

(d)

FIgURE 7: Antimicrobial activity of Ag NPs against marine pathogens. (a) The antibacterial activity of Ag NPs and Ag NPs-DNA conjugates against $V$. anguillus was determined by the Oxford cup method. The concentration of Ag NPs was $2.5 \mathrm{mM}$ and the concentration of DNA samples was $1 \mu \mathrm{M}$. Ultrapure water was used as negative control and $75 \%$ ethanol as positive control. (b) The antibacterial activity of different concentrations of Ag NPs against $V$. anguillus. (c) The effect of $2.5 \mathrm{mM} \mathrm{Ag} \mathrm{NPs} \mathrm{and} 1 \mu \mathrm{M}$ DNA or base on the growth curve of E. tarda. (d) Detection of the antibacterial effect of Ag NPs and Ag NPs-DNA conjugates on E. tarda using live bacteria counting method. The concentration of Ag NPs used in the experiment was $2.5 \mathrm{mM}$, and the concentration of poly-A sequence was $1 \mu \mathrm{M}$. 


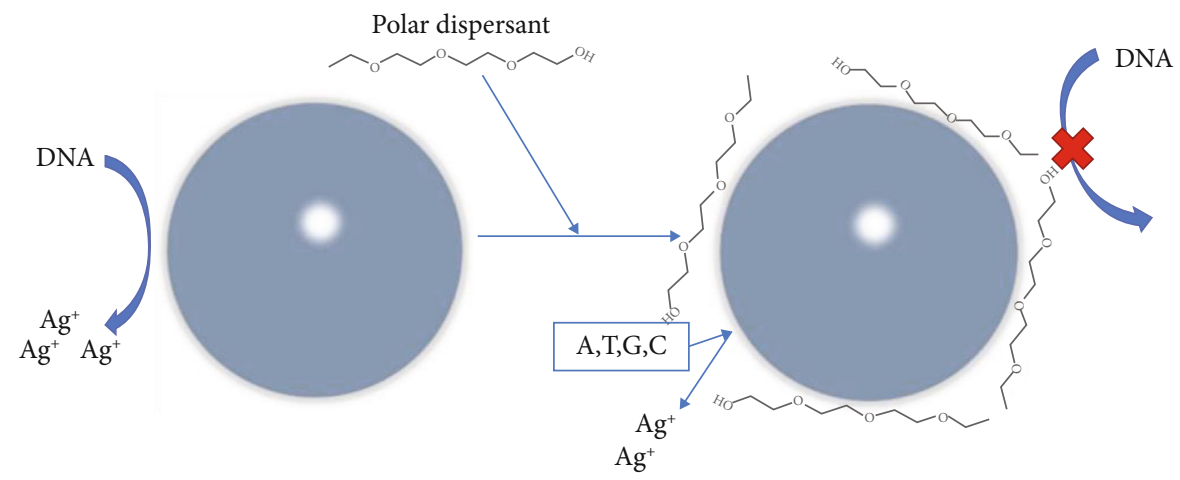

FIgURE 8: Schematic diagram of Ag NPs coated with polar dispersant and the interaction with DNA or bases.

As we all know, Ag NPs released silver ions with high biological activity in the antibacterial process, as they penetrated the cell barrier and destroyed the organisms $[22-24,28]$. We speculated that the existence of dispersants hindered the release of silver ions from Ag NPs, thus inhibiting the antibacterial activity.

In 2019, Zhang's research group reported that DNA has an etch effect on Ag NPs during the interaction between Ag NPs and DNA [25]. The silver ions were stripped from the surface of Ag NPs, thereby enhancing the resistance of Ag NPs to bacteria and tumors [25]. Comparing Ag NPs coated with triethylene glycol monoethyl ether, we speculate that Ag NPs were protected by the polar dispersant to avoid the etching effect by DNA. However, our experiments showed that the nucleotide bases still had an etching effect on Ag NPs. Figure 8 is a scheme of reaction between Ag NPs and DNA or nucleotide bases. In our study, the Ag NPs coated with polar materials still had the property of adsorption with biomacromolecules. Nevertheless, the antibacterial activity may be significantly reduced.

\section{Conclusions}

The study focused on the effects of properties of Ag NPs coated with polar material: dispersant triethylene glycol monoethyl ether. It could not prevent the interaction adsorption between Ag NPs and DNA. The fluorescence of FAMDNA still could be quenched by Ag NPs. The backbone of phosphate and bases played a main role in the adsorption. The adsorption and desorption experiments of DNA showed that the order of DNA functional groups on the interaction process is phosphate $>\mathrm{T}>\mathrm{C}>\mathrm{A}>\mathrm{G}$. The polar dispersant had a certain inhibitory effect on the antibacterial activity of $\mathrm{Ag}$ NPs. And small molecules such as bases could interact on the surface Ag NPs and release $\mathrm{Ag}^{+}$to perform the antibacterial activity. Ag NPs generally achieve antibacterial effects by releasing highly biologically active silver ions, but the Ag NP characteristic could be affected by polar materials that interacted with them. The antimicrobial activity was decreased significantly if Ag NPs were coated with polar materials, but the bases could increase the antimicrobial activity. It may be a way to control the antimicrobial activity of Ag NPs by adding an amount of bases.
This research provides strong theoretical support for the application of silver nanomaterials in the fields of antibacterial, biology, and medicine.

\section{Data Availability}

The interaction between Ag and DNA data used to support the findings of this study are included within the article and the supplementary information file. Also, all the data used to support the findings of this study are available from the corresponding author upon request.

\section{Conflicts of Interest}

The authors declare that there is no conflict of interest regarding the publication of this paper.

\section{Authors' Contributions}

Y. Feng was responsible for investigation, formal analysis, and writing of the draft; T. Deng was responsible for formal analysis and investigation; X. Lai was responsible for investigation and data curation; Z. Feng was responsible for resources; S. Wang was responsible for project administration; M. Lyu was responsible for supervision, writing, review, and editing. All authors have read and agreed to the published version of the manuscript. Yanli Feng and Tian Deng contributed equally to this work.

\section{Acknowledgments}

We are grateful to Prof. Juewen Liu's Lab of the University of Waterloo for the detection of TEM and fluorescence lifetime. This study was supported by the National Key Research and Development Program of China (2018YFC0311106), the Priority Academic Program Development of Jiangsu Higher Education Institutions (PAPD), and the Jiangsu Graduate Research Innovation Project KYCX18_2586 and KYCX19_ 2296.

\section{Supplementary Materials}

Figures S1-S5 and Table S1 are available online. (Supplementary Materials) 


\section{References}

[1] G. R. Tortella, O. Rubilar, N. Durán et al., "Silver nanoparticles: toxicity in model organisms as an overview of its hazard for human health and the environment," Journal of Hazardous Materials, vol. 390, no. 5, p. 121974, 2020.

[2] A. Feng, J. Cao, J. Wei, F. Chang, Y. Yang, and Z. Xiao, "Facile synthesis of silver nanoparticles with high antibacterial activity," Materials, vol. 11, no. 12, p. 2498, 2018.

[3] W. Salem, D. R. Leitner, F. G. Zingl et al., "Antibacterial activity of silver and zinc nanoparticles against Vibrio cholerae and enterotoxic Escherichia coli," International Journal of Medical Microbiology, vol. 305, no. 1, pp. 85-95, 2015.

[4] A. Panáček, M. Kolář, R. Večeřová et al., "Antifungal activity of silver nanoparticles against Candida spp," Biomaterials, vol. 30, no. 31, pp. 6333-6340, 2009.

[5] J. L. Elechiguerra, J. L. Burt, J. R. Morones et al., "Interaction of silver nanoparticles with HIV-1," Journal of Nanobiotechnology, vol. 3, no. 1, p. 6, 2005.

[6] S. Gurunathan, J. W. Han, D. N. Kwon, and J. H. Kim, "Enhanced antibacterial and anti-biofilm activities of silver nanoparticles against Gram-negative and Gram-positive bacteria," Nanoscale Research Letters, vol. 9, no. 1, p. 373, 2014.

[7] D. Archana, B. K. Singh, J. Dutta, and P. K. Dutta, "ChitosanPVP-nano silver oxide wound dressing: in vitro and in vivo evaluation," International Journal of Biological Macromolecules, vol. 73, no. 2, pp. 49-57, 2015.

[8] H. Agarwal, A. K. Gupta, N. Gupta, S. Mukharjee, and C. K. Durga, "Comparison of results of silver-impregnated dressing with povidone iodine based-dressing in patients with diabetic foot," Hellenic Journal of Surgery, vol. 87, no. 6, pp. 465-467, 2015.

[9] D. Huo, J. He, H. Li et al., "Fabrication of Au@Ag core-shell NPs as enhanced CT contrast agents with broad antibacterial properties," Colloids \& Surfaces B Biointerfaces, vol. 117, no. 5, pp. 29-35, 2014.

[10] X. Chen and H. J. Schluesener, "Nanosilver: a nanoproduct in medical application," Toxicology Letters, vol. 176, no. 1, pp. 112, 2008.

[11] K. Hosoyama, M. Ahumada, C. D. McTiernan et al., "Multifunctional thermo-crosslinkable collagen-metal nanoparticle composites for tissue regeneration: nanosilver vs. nanogold," RSC Advances, vol. 7, no. 75, pp. 47704-47708, 2017.

[12] J. C. Hannon, J. P. Kerry, M. Cruz-Romero, S. Azlin-Hasim, and E. Cummins, "An assessment of the migration potential of nanosilver from nanoparticle coated low density polyethylene food packaging into food simulants," Food Additives \& Contaminants. Part A, Chemistry, Analysis, Control, Exposure \& Risk Assessment, vol. 33, no. 1, pp. 167-178, 2015.

[13] N. V. K. Thanh and N. T. P. Phong, "Investigation of antibacterial activity of cotton fabric incorporating nano silver colloid," Journal of Physics: Conference Series, vol. 187, no. 1, p. 012072, 2009.

[14] S. H. Jeong, Y. H. Hwang, and S. C. Yi, "Antibacterial properties of padded PP/PE nonwovens incorporating nano-sized silver colloids," Journal of Materials Science, vol. 40, no. 20, pp. 5413-5418, 2005.

[15] Y. W. Yang and J. L. Liu, "Latest advances in the researches in and application of nanoparticle silver," Industrial Catalysis, vol. 271, no. 2, pp. 343-350, 2003.

[16] P. Prasher, M. Sharma, H. Mudila et al., "Emerging trends in clinical implications of bio-conjugated silver nanoparticles in drug delivery," Colloid and Interface Science Communications, vol. 35, no. 3, p. 100244, 2020.

[17] M. Rahimi, E. B. Noruzi, E. Sheykhsaran et al., "Carbohydrate polymer-based silver nanocomposites: recent progress in the antimicrobial wound dressings," Carbohydrate Polymers, vol. 231, p. 115696, 2020.

[18] T. T. Zhao, Q. Y. Chen, and H. Yang, "Spectroscopic study on the formation of DNA-Ag clusters and its application in temperature sensitive vehicles of DOX," Spectrochimica Acta Part A Molecular \& Biomolecular Spectroscopy, vol. 137, pp. 66-69, 2015.

[19] J. C. Trefry, J. L. Monahan, K. M. Weaver et al., "Size selection and concentration of silver nanoparticles by tangential flow ultrafiltration for SERS-based biosensors," Journal of the American Chemical Society, vol. 132, no. 32, pp. 1097010972, 2010.

[20] J. Willem de Vries, S. Schnichels, J. Hurst et al., "DNA nanoparticles for ophthalmic drug delivery," Biomaterials, vol. 157, pp. 98-106, 2018.

[21] Q. Pan, J. Zhang, X. Li et al., "Construction of novel multifunctional luminescent nanoparticles based on DNA bridging and their inhibitory effect on tumor growth," RSC Advances, vol. 9, no. 26, pp. 15042-15052, 2019.

[22] N. Kasyanenko, V. Bakulev, I. Perevyazko et al., "Model system for multifunctional delivery nanoplatforms based on DNApolymer complexes containing silver nanoparticles and fluorescent dye," Journal of Biotechnology, vol. 236, pp. 78-87, 2016.

[23] X. Xu, J. Wang, F. Yang, K. Jiao, and X. Yang, "Label-free colorimetric detection of small molecules utilizing DNA oligonucleotides and silver nanoparticles," Small, vol. 5, no. 23, pp. 2669-2672, 2009.

[24] J. Fu, Z. Zhang, and G. Li, "Progress on the development of DNA-mediated metal nanomaterials for environmental and biological analysis," Chinese Chemical Letters, vol. 30, no. 2, pp. 285-291, 2019.

[25] E. Zhang, S. Fu, R. X. Wang et al., "Role of Cu element in biomedical metal alloy design," Rare Metals, vol. 38, no. 6, pp. 476-494, 2019.

[26] J. Zhang, Y. H. Sun, Y. Zhao et al., "Antibacterial ability and cytocompatibility of $\mathrm{Cu}$-incorporated $\mathrm{Ni}-\mathrm{Ti}-\mathrm{O}$ nanopores on NiTi alloy," Rare Metals, vol. 38, no. 6, pp. 552-560, 2019.

[27] B. Huang, X. Liu, L. Tan et al., “"Imitative” click chemistry to form a sticking xerogel for the portable therapy of bacteriainfected wounds," Biomaterials Science, vol. 7, no. 12, pp. 5383-5387, 2019.

[28] Y. Luo, J. Li, X. Liu et al., "Dual metal-organic framework heterointerface," ACS Central Science, vol. 5, no. 9, pp. 15911601, 2019.

[29] Y. Li, X. Liu, L. Tan et al., "Rapid sterilization and accelerated wound healing using $\mathrm{Zn} 2$ +and graphene oxide modified gC3N4under dual light irradiation," Advanced Functional Materials, vol. 28, no. 30, pp. 1800299.1-1800299.12, 2018.

[30] S. Hu, T. Yi, Z. Huang et al., "Etching silver nanoparticles using DNA," Materials Horizons, vol. 6, no. 1, pp. 155-159, 2019.

[31] V. K. Sharma, K. M. Siskova, R. Zboril, and J. L. Gardea-Torresdey, "Organic-coated silver nanoparticles in biological and environmental conditions: fate, stability and toxicity," Advances in Colloid and Interface Science, vol. 204, pp. 15-34, 2014. 
[32] A. Ghosh, N. Karedla, J. C. Thiele, I. Gregor, and J. Enderlein, "Fluorescence lifetime correlation spectroscopy: basics and applications," Methods, vol. 140-141, pp. 32-39, 2018.

[33] X. Zhang, M. R. Servos, and J. Liu, "Fast pH-assisted functionalization of silver nanoparticles with monothiolated DNA," Chemical Communications, vol. 48, no. 81, pp. 10114-10116, 2012.

[34] M. Guzman, J. Dille, and S. Godet, "Synthesis and antibacterial activity of silver nanoparticles against gram-positive and gram-negative bacteria," Nanomedicine: Nanotechnology, Biology and Medicine, vol. 8, no. 1, pp. 37-45, 2012. 